\title{
Relations entre la durée du cycle nymphal et le poids originel de la pupe ( $G$. morsitans).
}

\author{
par L. MAILLOT
}

\begin{abstract}
RESUME
L'auteur établit qu'il existe chez $G$, morsitans d'élevage une corrélation faible mais significative dans les deux sexes entre le poids de la pupe et la durée du cycle nymphal en groupant quatre séries d'observations (femelles : $\mathrm{r}=0,52$, mâles $=0,43: \mathrm{P}<0,01$ ).

Ces résultats, qui gagneraient à être précisés par un plus grand nombre d'observations, semblent toutefois Jaisser présumer que la température n'est peut-être pas l'unique agent causal des variations saisonnières de la durée du cycle nymphal observées chez la tsé-tsé dans la nature.
\end{abstract}

L'auteur, ayant constaté que la durée du cycle nymphal semblait en relation avec le poids de la mouche à l'éclosion et également avec le poids originel de la pupe, a entrepris différentes séries d'observations pour vérifier le bien-fondé de cette hypothèse.

En 1967 un très petit nombre d'observations laissait supposer qu'il pouvait exister une concordance entre poids de la mouche à l'éclosion et durée du cycle nymphal, par ailleurs à la lecture des registres tenus au laboratoire où en septembre 1967 était inscrite une série de pesées de pupes avec indication des dates de ponte, d'éclosion et du sexe de l'adulte, la même hypothèse semblait pouvoir être retenue pour Ie poids de la pupe.

Aussi trois séries d'expériences ont été entreprises dans ce sens, deux en mai 1968, une en juin 1969. Un certain nombre de pupes ont été recueillies à une date déterminée le plus tôt possible après la ponte et pesées à la balance Sartorius, on note la date de l'éclosion, le sexe de l'adulte, qui est pesé peu après l'éclosion. Pour chaque période nymphale dans chaque sexe on a établi le poids moyen des pupes et celui des adultes.

- Il est évident que c'est le poids de la pupe qui est susceptible d'influer sur la durée du cycle nymphal et non l'inverse - mais dans chaque groupe d'observations, il est préférable de comparer les poids moyens des pupes de différents cycles échelonnés de 27 à 35 jours que de comparer les cycles nymphaux moyens des poids de pupes variant de 15 à 38 milligrammes, car on obtient ainsi des groupes plus étendus dont la comparaison a permis plus facilement de prévoir une corrélation entre les deux variables. Corrélation en général ne signifie pas qu'entre deux variables il y ait obligatoirement un rapport de cause à effet (6) mais dans ce cas particulier il paraît logique de supposer que c'est le poids de la pupe qui semble avoir une influence sur la durée du cycle nymphal (voir plus loin).

Les chiffres obtenus sont résumés dans l'appendice ci-joint.

\section{OBSERVATIONS}

De tous ces chiffres se dégagent en général les constatations suivantes :

— le poids moyen originel des pupes mâles et des pupes femelles n'est pas sensiblement différent;

- le poids moyen de l'adulte femelle à 
l'ćclosion est le plus souvent supérieur (sauf série C).

- Dans la plupart des séries le poids moyen des pupes d'un cycle nymphal donné est dautant plus élevé que le cycle nymphal correspondant est plus long (voir remarque plus haut).

Dans la dernière série, série D de juin 1969, où le poids moyen des pupes est nettement plus élevé que dans les autres séries, les durées des cycles nymphaux sont plus longues.

La diminution du poids de la pupe à l'adulte (cf, 1)

1. est en gênéral plus forte chez le mâle (voir plus haut);

2. dans un tiers des cas, d'autant plus accentuée que le cycle nymphal est plus long.

- les températures relevées (températures moyennes pendant tout le cycle et températures moyennes relevées dans les trois jours qui précèdent l'éclosion), ne sont guère différentes d'une série à l'autre sauf dans la $1^{\text {re }}$ série $\mathrm{A}$ où la température moyenne générale atteignait $24,5^{\circ} \mathrm{C}$.

\section{DISCUSSION}

Différentes analyses statistiques pratiquées dans chacune des séries n'ont révélé aucune corrélation significative.

Mais si l'on groupe tous les résultats des quatre séries dans un tableau de corrélation, entre poids de la pupe et durée du cycle nymphal et qu'à partir de ces résultats se calculent les coefficients de corrélation entre les deux valeurs, on trouve chez les femelles un coefficient de corrélation de 0,52 et chez les mâles de 0,43 . Les deux coefficients sont significatifs avec une probabilité de 0,01 .

Ce qui implique que la corrélation est valable grosso modo 1 fois sur 4 chez les femelles et 1 fois sur 5 chez les mâles (femelles $r^{2}=0,27$, mâles $\mathrm{r}^{2}=0,18$ ).

Il est admis classiquement (4) que la température a un rôle sur la durée du cycle nymphal qui s'allonge quand les températures s'abaissent

(4)

$$
\mathrm{CN}=\frac{1}{0,0323+0,0028(\mathrm{t}-24)}
$$

et vice-versa, ici les températures enregistrées ne peuvent suffire à expliquer les variations de durée du cycle nymphal d'une série à une autre.

\section{CONCLUSION}

La prédominance de certains résultats, l'existence de coefficients de corrélation, faibles mais significatifs, permettent de présumer que la durée du cycle nymphal est en partie dépendante du poids originel de la pupe et, s'il n'est pas exclu qu'un plus grand nombre d'observations ne parviennent à confirmer plus nettement ces hypothèses, les particularités observées méritaient cependant d'être signalées.

\section{APPENDICE}

- $\mathbf{P}$ : poids moyen des pupes dans la catégorie étudiée;

- pour l'ensemble d'une série :

$\mathbf{P}$ est le poids moyen de toutes les pupes, $\mathrm{P}^{\prime}$ celui des pupes écloses;

- A : poids moyen des mouches adultes dans la catégorie étudiée (à ou peu après l'éclosion);

- A/P : rapport des deux poids moyens correspondants;

- $\mathrm{CN}$ : durée moyenne du cycle nymphal;

- CN 30, CN 31, etc. : catégorie correspondant à un cycle de 30,31 jours etc.;

- nb: nombre d'observations;

- $\mathrm{T}$ : température moyenne.

\section{A. Registre du laboratoire}

Ponte du 15-9-1967

123 pupes :

119 éclosions dont 64 mâles et 55 femelles

$\mathrm{P}=26,94 \mathrm{mg} \quad \mathbf{P}^{\prime}=26,92$

Mâles $\mathrm{P}=26,98 \mathrm{mg} \quad \mathrm{CN}=31,32$ jours

$\mathrm{CN} 30: \mathrm{nb}=7 \quad \mathrm{P}=23,03$

CN 31: $\mathbf{n b}=35 \quad \mathrm{P}=26,44$

$\mathrm{CN} 32: \mathrm{nb}=22 \quad \mathrm{P}=29,10$

Femelles $\quad \mathrm{P}=26,84 \quad \mathrm{CN}=28,8$

CN 27: $\mathrm{nb}=4 \quad \mathrm{P}=21,21$

CN 28: nb $=8 \quad \mathrm{P}=23,90$

$\mathrm{CN} 29: \mathrm{nb}=36 \quad \mathrm{P}=27,51$

$\mathrm{CN} 30: \mathrm{nb}=7 \quad \mathrm{P}=29,56$

\section{Températures}

du $15-9$ au $17-10, T=24,5^{\circ} \mathrm{C}$

(à $4 \mathrm{~h}, \mathrm{~T}=24,2$; à $12 \mathrm{~h}, 24,8$; à $20 \mathrm{~h}, 24,6$ )

3 jours ayant l'éclosion pour tous les cycles chez les femelles, $\mathrm{T}=24,9{ }^{\circ} \mathrm{C}$; chez les mâles, $\mathrm{T}=24,4^{\circ} \mathrm{C}$. 


\section{B. Pontes du 14-5-1968}

54 pupes:

54 éclosions dont 31 mâles et 23 femelles $\mathrm{P}=\mathrm{P}^{\prime}=26,73 \mathrm{mg}$

Mâles $\mathrm{P}=26,77 \quad \mathrm{~A}=18,81 \quad \mathrm{CN}=31,7$

CN 31 nb $13 \quad$ P 25,70 A $18.12 \quad$ A/P 0,70

CN 32 nb 15 P 27,27 A 19,28 A/P 0,70

$\mathrm{CN} 33$ nb 3 P 28,38 A 19,46 A/P 0,65

Femelles $\mathrm{P}=26,68 \quad \mathrm{~A}=20,63 \quad \mathrm{CN}=29,3$

CN 28 nb 2 P 22,22 A 16,53 A/P 0,74

CN 29 nb 13 P 25,01 A 19.58 A/P 0,77

CN 30 nb 8 P 30,49 A 23,62 A/P 0,77

\section{Températures}

du $14-5$ au 16-6 $\mathrm{T}=24,9^{\circ} \mathrm{C}$

(à $4 \mathrm{~h}, 24,6$; à $12 \mathrm{~h}, 25,3$; à $20 \mathrm{~h}, 24,9$ )

3 jours avant l'éclosion dans tous les cycles chez les femelles, $\mathrm{T}=25,1^{\prime \prime} \mathrm{C}$; chez les mâles $\mathrm{T}=25,7^{\circ} \mathrm{C}$.

\section{Pontes du 21-5-1968 \\ 59 pupes: \\ 51 éclosions dont 27 mâles et 24 femelles \\ $\mathrm{P}=25.68 \mathrm{mg} \quad \mathrm{P}^{\prime}=25,59$}

Males $\mathrm{P}=25,71 \mathrm{mg} \quad \mathrm{A}=19,34 \quad \mathrm{CN}=31,1$ jours

$\mathrm{CN} 30 \quad \mathrm{nb} \quad 2 \quad \mathrm{P} 23,83$ A $16,93 \quad \mathrm{~A} / \mathrm{P}=0,71$

CN 31 nb $21 \quad \mathrm{P} 25,71$ A $19,37 \quad \mathrm{~A} / \mathrm{P}=0,75$

CN 32 nb 4 P 26,64 A 20,44 A/P $=0,77$
Fenelles $\mathbf{P}=25,46 \quad \mathrm{~A}=19,16 \quad \mathrm{CN}=28,9$

CN 28 nb 4 P 21,23 A 16,25 A/P $=0,78$

CN 29 nb $19 \quad \mathrm{P} 26,45$ A $19,82 \quad \mathrm{~A} / \mathrm{P}=0,75$

$\mathrm{CN} 30 \mathrm{nb} \quad 1 \quad \mathrm{P} 25,45$ A $18,72 \quad \mathrm{~A} / \mathrm{P}=0,75$

\section{Températures}

du $21-5$ au 23-6 $\mathrm{T}=25,3^{\circ} \mathrm{C}$

(à 4 h. 25,0; à 12 h, 25,7; à $20 \mathrm{~h}, 25,3$ )

3 jours avant l'éclosion dans tous les cycles pour les femelles $\mathrm{T}=25,9^{\prime \prime} \mathrm{C}$; pour les mâles $\mathrm{T}=25,8^{\circ} \mathrm{C}$.

D. Pontes du 24 et 25-6-1969

87 pupes:

85 éclosions dont 44 mâles et 41 femelles $\mathrm{P}=29,36 \quad \mathrm{P}^{\prime}=29,37$

\begin{tabular}{|c|c|c|c|c|}
\hline Mâles & $P=2$ & $94 \mathrm{mg}$ & $A=20$ & $\mathrm{CN}=33$ \\
\hline $\mathrm{CN} 32$ & $\mathrm{nb}$ & P 26,79 & A 19,88 & $\mathrm{~A} / \mathrm{P}=0,74$ \\
\hline $\mathrm{CN} 33$ & nb 14 & P 26,63 & A 19.06 & $\mathrm{~A} / \mathrm{P}=0,71$ \\
\hline $\begin{array}{l}\text { CN } 33 \\
\text { à } 34\end{array}$ & nb 6 & P 30,19 & A 21.32 & $\mathrm{~A} / \mathrm{P}=0,71$ \\
\hline N 34 & no & P 30,63 & A 21,79 & $A / P=0,71$ \\
\hline $\mathrm{CN} 34$ & $\mathrm{nb}$ & P 34,33 & A 23,55 & $\mathrm{~A} / \mathrm{P}=0,76$ \\
\hline
\end{tabular}

Fenelles $\mathrm{P}=29,83 \mathrm{mg} \quad \mathrm{A}=23,47 \quad \mathrm{CN}=30,9$

CN 30 nb $13 \quad$ P 29,21 A 23,26 A/P $=0,80$

CN 31 nb $21 \quad$ P $29,45 \quad$ A $23,15 \quad \mathrm{~A} / \mathrm{P}=0,78$

CN 32 nb 7 P 32,15 A $28,84 \quad$ A/P $=0,77$

\section{Températures}

du 24-6 au 29-7-1969 $\mathrm{T}=25,0^{\circ} \mathrm{C}$

(à 4 h. 25,0 ; à $12 \mathrm{~h}, 24,9$; à $20 \mathrm{~h}, 25,0$ )

3 jours avant l'éclosion dans tous les cycles

pour les femelles $\mathrm{T}=25,2^{\circ} \mathrm{C}$; pour les mâles $\mathbf{T}=$ $25,9 * \mathrm{C}$.

\section{SUMMARY}

\section{Relation between weight of puparia and length of puparial stage}

The author shows there is a light but significant correlation between weight of puparia and time of puparial life in both sexes of laboratorybred $G$. morsitans by gathering four sets of data (females: $\mathbf{r}=0,52$; males : $r=0,43 ; \mathrm{P}<0,01)$.

These results would be nore valuable if confirmed by more numerous data, but however presumably suggest that temperature is not the single factor acting upon the seasonal differences of puparial stage under field conditions.

\section{RESUMEN}

Relaciones entre la duración del ciclo ninfal y el peso de origen de Ia pupa $G$. moisituns

El autor establece que existe, en $G$. morsitans de cria, una correlación poco importante pero significativa en los dos sexos entre el peso de la pupa y la duración del ciclo ninfal al agrupar cuatro series de observaciones (hembras : $\mathrm{r}=0,52$; machos $=0,43$; $\mathrm{P}<0,01$ ).

Dichos resultados que ganarian con ser precisados por un más gran número de observaciones, sin embargo parecen presuponer que la temperatura acaso no es el único agente causal de las variaciones estacionales de la duración del ciclo ninfal observadas en la tsetse en la natura. 


\section{BIBIJOGRAPHIE}

1. BURSELL (E.), «L'équilibre hydrique des pupes de tsé-tsé » (The water balance of tsetse pupae), Philos. Trans. R. Soc. (B), 1958, 241 (680) : 179-210.

2. BUXTON (P.A.), «L'histoire naturelle des tsé-tsé 》 (The natural history of tsetse flies), London, H. K. Lewis, 1955. Pp. 382-389.

3. CHORLEY (J. K.), "Biologie de Glossina morsitans dans la zone à mouches d'Umniati» (The bionomies of Glossina morsitans in the Umniati fly belt, Southern Rhodesia), Bull. ent. Res. 1929, 20: 279-301.
4. JACKSON (C. H, N), «La biologie des tsé-tsé 》 (The biology of tsetse flies), Biol. Rev. 1949, 24 : 174-199.

5. LLOYD (LI.), «Notes sur Glossina morsitans Westw. dans la vallée de la Luangwa en Rhodésie du Nord" (Notes on Glossina morsitans Westw. in the Luangwa valley, Northern Rhodesia), Bull. ent. Res. 1912, 3 : 233-239.

6. SCHREIDER (E.), «La biométrie», Paris, Presses Universitaires de France, 1960 (Collection \&Que sais-je? »), 871. 